\title{
Absenteísmo de usuários como fator de desperdício: desafio para sustentabilidade em sistema universal de saúde
}

\author{
Non-attendance as a factor of waste: challenge for SUStainability in \\ a universal health system
}

Sonia Maria Beltrame', Adauto Emmerich Oliveira', Maria Angelica Borges dos Santos ${ }^{\mathbf{2}}$, Edson

Theodoro Santos Neto 1

DOI: $10.1590 / 0103-1104201912303$

RESUMO O absenteísmo de usuários em consultas e exames é considerado um problema mundial na assistência à saúde, gerando desperdício de recursos tanto no setor público como no setor privado. $\mathrm{O}$ objetivo deste estudo foi o de estimar o desperdício de recursos monetários vinculado ao absenteísmo em procedimentos especializados no Sistema Único de Saúde (SUS) na Região de Saúde Metropolitana do Espírito Santo (RSM-ES) entre os anos de 2014 e 2016. Analisaram-se 1.002.719 procedimentos, sendo 666.182 consultas e 336.537 exames especializados. Os dados de absenteísmo foram retirados dos registros administrativos do Sistema de Regulação do ES (SisReg-ES), fornecidos pela Secretaria Estadual de Saúde. Os valores monetários foram obtidos por meio da Tabela SUS, da tabela complementar de convênios e da tabela de custos estimados, segundo o tipo de prestador envolvido no atendimento. A taxa média de absenteísmo para consultas foi de $38,6 \%$ (257.025 consultas), gerando um total estimado de $\mathrm{R} \$ 3.558 .837,88$; e para exames especializados, foi de $32,1 \%$ (108.103 exames), em um total estimado de $\mathrm{R} \$ 15.007 .624,15$. Os valores totais desperdiçados são significativos e evidenciam o desafio constante na agenda dos gestores na busca pela SUStentabilidade em sistemas universais de saúde.

PALAVRAS-CHAVE Absenteísmo. Atenção secundária à saúde. Gestão em saúde. Integralidade em saúde.

1 Universidade Federal do Espírito Santo (Ufes) Vitória (ES), Brasil. sonia_beltrame@yahoo. combr

2 Fundação Oswaldo Cruz (Fiocruz), Escola Nacional de Saúde Pública Sergio Arouca (Ensp) - Rio de Janeiro (RJ), Brasil.

\begin{abstract}
Non-attendance to specialized healthcare appointments, diagnostic, and therapeutic procedures is a global problem in healthcare, leading to the wastage of resources both in the public and private sectors. This study aims to estimate financial wastage of resources linked to non-attendance to scheduled health specialist appointments and procedures in the metropolitan region of the Espírito Santo State (ES), Brazil, between 2014 and 2016. We studied 1.002.719 specialized healthcare procedures, being 666.182 appointments with specialized physicians and other healthcare professionals, and 336.537 specialized diagnostic and other therapeutic procedures. Non-attendance date were retrieved from the administrative procedure scheduling database (SisReg-ES) available in the ES Regulatory Agency and provided by the ES Health Office. Financial values used to estimate wastage were retrieved from the SUS and other standard tariff lists for medical procedures, and used according to the administrative regime of the three types of service providers. Nonattendance average rate for medical and health specialists' appointments achieved $38.6 \%$, or 257.025 missed appointments, equivalent to an estimated waste of resources of $R \$ 3.558 .837,88$. Non-attendance as for other specialized procedures reached $32.1 \%$, or 108.103 missed procedures, equivalent to $R \$ 15.007 .624,15$. Total wasted resources reached significant values, evincing the ongoing challenge to managers seeking to attain SUStainable universal health care systems.
\end{abstract}

KEYWORDS Absenteeism. Secondary care. Health management. Integrality in health. 


\section{Introdução}

O absenteísmo de usuários é o ato de não comparecer às consultas e/ou aos procedimentos agendados sem qualquer comunicação prévia ao local de realização' 1 É considerado um problema mundial na assistência à saúde tanto no setor público como no privado, de acordo com trabalhos publicados no Brasil2,3 e no mundo ${ }^{4-6}$. Uma revisão sistemática sobre o tema apontou uma taxa média mundial de absenteísmo de $23 \%$, sendo os maiores índices encontrados na África (43,0\%), seguido pela América do Sul (27,8\%), Ásia (25,1\%), América do Norte (23,5\%), Europa (19,3\%) e Oceania $(13,2 \%)^{7}$. No Sistema Único de Saúde (SUS), o absenteísmo revela-se um problema crônico, com taxas próximas ou superior a $25 \%^{8}$ atingindo percentuais altos em diversos tipos de atendimento e especialidades médicas ${ }^{9}$.

O absenteísmo foi associado a algumas causas, como esquecimento ${ }^{3,10}$, falhas na comunicação entre o serviço e o usuário, melhora dos sintomas de adoecimento ${ }^{11}$, agendamento em horário de trabalho ${ }^{2,10-12}$, falta de transporte e dia da semana agendado ${ }^{4}$. Entre as suas consequências, destacam-se o aumento da fila de espera e de demandas por urgência 2,12, o desperdício de recursos públicos ${ }^{2,4-6,13}$, a redução da produtividade e perda da eficiência da clínica e da gestão ${ }^{5}$. Essas consequências comprometem o acesso e acarretam aumento dos custos com a assistência à saúde ${ }^{2,3,14}$, gerando custos sociais ${ }^{15}$, estimulando atitudes negativas no profissional ${ }^{16} \mathrm{e}$ acarretando atraso no diagnóstico e tratamento adequado ${ }^{13}$. Em síntese, o absenteísmo é considerado um fenômeno multicausal, repercutindo em todos os envolvidos: gestão, trabalhador e usuário ${ }^{2,3}$.

A assistência à saúde no SUS é dividida em atenção básica, atenção secundária ou média complexidade e atenção terciária ou alta complexidade ${ }^{17}$. A porta de entrada por iniciativa própria do usuário ao sistema é pela atenção básica, por meio das Unidades Básicas de Saúde (UBS) ou de uma Unidade de Pronto Atendimento (UPA) ${ }^{17,18}$. A atenção básica deve ser resolutiva em mais de $85 \%$ dos problemas de saúde da população, e o usuário deve ser encaminhado para a atenção especializada quando for necessário ${ }^{19}$.

Entretanto, desde a criação do SUS, em 1988, o sistema vem enfrentando inúmeras dificuldades quanto ao acesso do cidadão às ações e serviços de saúde resolutivos, adequados, oportunos e eficazes. É inegável a extensão de benefícios trazidos à população ao longo da sua existência, mas, na prática, ainda é parcial, com inúmeras debilidades e insuficiências ${ }^{20}$. O SUS foi implantado, mas não foi consolidado ${ }^{21}$. O acesso aos serviços especializados ou de média complexidade tem sido destacado como um dos principais obstáculos para a sua efetivação ${ }^{\mathbf{2 2}}$.

O envelhecimento da população tem contribuído para aumentar as Doenças Crônicas Não Transmissíveis (DCNT), as demandas na atenção especializada, portanto, impõe novos desafios ao sistema de saúde ${ }^{\mathbf{1 8}}$. O aumento da prevalência das DCNT pode também ser explicado pelo maior acesso aos serviços de saúde e meios de diagnósticos dessas doenças ${ }^{23}$. Atualmente, a demanda por procedimentos diagnósticos e terapêuticos em decorrência das DCNT está se tornando prioridade em saúde pública no Brasil24.

Nesse contexto, a gestão dos serviços precisa considerar, para o seu planejamento, as mudanças no cenário de adoecimento do País nas últimas décadas ${ }^{25}$. Assim, para qualificar a gestão dos serviços, em 2008, o Ministério da Saúde (MS), instituiu a Política Nacional de Regulação em Saúde ${ }^{26}$. A regulação do acesso à saúde é utilizada pelo Estado como um importante instrumento de gestão pública, ainda em processo de aprimoramento, com o objetivo de alcançar a eficiência, a equidade e o equilíbrio entre a oferta, demanda e financiamento ${ }^{27}$. O sistema de regulação (SisReg) utilizado é on-line e disponibilizado pelo MS, pelo Departamento de Informática do SUS (Datasus), para gerenciar todo complexo regulatório e operar as centrais de regulação no País. Sob esse aspecto, a política de regulação permite aos gestores conhecer o tamanho 
das filas de espera e do absenteísmo por meio do banco de dados que produz, orientando a gestão na busca de soluções.

Na Região de Saúde Metropolitana do Espírito Santo (RSM-ES), o absenteísmo na atenção especializada é um problema frequente verificado pela Secretaria Estadual de Saúde (Sesa-ES) e tem ocasionado prejuízos à população e aos cofres públicos, considerando que é grande o número de usuários do SUS com consultas e exames agendados nas diversas especialidades que não comparecem e não desmarcam. Segundo os dados da Sesa-ES, no primeiro semestre de 2015, a taxa de absenteísmo foi de $38 \%$, para consultas e exames especializados agendados pela Central de Regulação28.

Assim, o absenteísmo na atenção especializada tem chamado a atenção dos gestores do SUS no Brasil, porque contribui para o aumento da fila de espera no atendimento, somado aos pacientes faltosos que tendem a voltar para a fila, o que acarreta diminuição do aproveitamento da oferta, que, por conseguinte, aumenta o tempo de espera para um novo agendamento ${ }^{2}$. Atualmente, os desafios para a oferta de serviços na atenção especializada são inúmeros e, possivelmente, serão agravados pelas consequências da Emenda Constitucional (EC) 95/2016, que limita os gastos públicos por 20 anos e pode dificultar o incremento de recursos para a saúde e demais políticas sociais, representando uma grande ameaça a essas demandas. Os cortes orçamentários poderão repercutir nos indicadores de saúde da população 29,30 .

Nesse sentido, aperfeiçoar a gestão na saúde é necessário para combater e reduzir o desperdício e aumentar a eficiência, otimizando os recursos financeiros disponíveis. A Organização Mundial da Saúde (OMS), em 2010, no relatório 'Financiamento da Cobertura Universal', revela que de $20 \%$ a $40 \%$ de todos os gastos em saúde são desperdiçados por ineficiência, recursos estes que poderiam ser redirecionados para atingir a cobertura universal.

Por outro lado, são escassos estudos que abordem o absenteísmo como fonte de desperdício e apliquem avaliação econômica e métodos analíticos dos serviços não executados evidenciando o desperdício que ele causa ${ }^{4}$. As publicações, em sua maioria, abordam as causas e estratégias para sua redução, sendo enfáticas ao afirmar que causam prejuízos monetários, geralmente com abordagens apoiadas em evidências empíricas ${ }^{5}$. Assim, cada vez mais, torna-se necessário que os conceitos de avaliação econômica façam parte da rotina dos sistemas e serviços de saúde ${ }^{\mathbf{3} 1}$.

Com base nessas considerações, identificando o absenteísmo como um problema crescente no SUS, o objetivo deste estudo foi estimar o desperdício de recursos monetários vinculado ao absenteísmo em procedimentos especializados no SUS na RSM-ES entre os anos de 2014 e 2016.

\section{Métodos}

Trata-se de um estudo descritivo baseado em dados de registros administrativos, conduzido na RSM-ES, localizada na região Sudeste. A região é composta por 20 municípios que apresentam os melhores Índices de Desenvolvimento Humano Municipal (IDH-M) do estado $(0,68$ a 0,856) e população de 1.935.393 habitantes ${ }^{32}$.

Foram utilizados dados gerados de 1.002.719 procedimentos de consultas e exames especializados, agendados de janeiro a dezembro de 2014 a 2016 no SisReg-ES, fornecidos pela SesaES. Os procedimentos agendados referem-se às vagas disponibilizadas por especialidades pela Programação Pactuada Integrada (PPI), realizada entre estado e municípios da região. Foram analisados 666.182 consultas especializadas e 336.537 exames especializados, em um total de 38 locais executantes, que incluíram participações variadas por especialidade de agendamentos em estabelecimentos públicos (gestão direta) e contratados do SUS, estabelecimentos filantrópicos e serviços públicos com gestão de Organizações Sociais (OS).

As variáveis selecionadas foram: ano (2014, 2015 e 2016); procedimento agendado (códigos 
para tipos de consulta e exame especializado agendados); procedimento confirmado (realizado com o comparecimento do paciente); procedimento não confirmado (absenteísmo devido ao não comparecimento do paciente); consultas especializadas e exames especializados (rol de procedimentos da atenção de média complexidade); e local executante (locais de referência, onde os procedimentos foram realizados). Inicialmente, os dados foram separados segundo consultas e exames e ano de agendamento.

As consultas foram registradas segundo diversas nominações usadas pelo SisReg-ES, tendo sido necessário utilizar a Classificação Brasileira de Ocupações (CBO), do Ministério do Trabalho, para recodificar a especialidade médica e outras do profissional executante. As diversas nominações para a mesma especialidade foram agrupadas em uma única classificação da CBO para posterior codificação e definição de correspondência (de-para) com os procedimentos das Tabelas de valores /preços utilizados.

Os exames especializados codificados pela tabela do SisReg-ES também foram recodificados, a exemplo do procedimento adotado para as consultas. Os exames foram agrupados em grupos e subgrupos, conforme classificação da Tabela Unificada de Procedimentos, Medicamentos e Insumos Estratégicos do SUS (Tabela SUS), identificando-se o número de exames agendados, exames confirmados, absenteísmo e o montante monetário. $\mathrm{O}$ grupo 02 foi composto por todos os procedimentos dos subgrupos com finalidade diagnóstica; o grupo 03, por todos os procedimentos dos subgrupos com exames relacionados com os procedimentos clínicos; e o grupo 04, por todos os procedimentos dos subgrupos com exames relacionados com os procedimentos cirúrgicos.

Os valores atribuídos a cada procedimento variaram segundo as remunerações vinculadas aos locais executantes e respectivas naturezas administrativas. Para procedimentos efetuados nos estabelecimentos públicos estadual e federal (gestão direta) e contratados do SUS (gestão indireta), a referência para valores de procedimentos foi a Tabela SUS (Tabela SUS/2018). Para os filantrópicos, foram somados os valores das tabelas complementares dos aditivos de 2018 fornecidas pela Sesa-ES e os valores da Tabela SUS/2018. Para os serviços públicos com gestão de OS, foi utilizada tabela de custos estimados por local executante, fornecida pela Sesa que adota a ferramenta denominada Key Performance Indicators for Health (KPIH) para efetuar o sistema de gestão de custos. Todos os valores foram atualizados para o ano de 2018.

Procedimentos de consultas/exames foram agrupados por especialidades, quantificando-se o total agendado, total confirmado, absenteísmo (procedimentos não confirmados $=$ agendados - confirmados) e o valor monetário anual por especialidade atribuído ao absenteísmo, segundo as tabelas por natureza administrativas do local executante. Posteriormente, foram elencadas as especialidades em ordem crescente pela taxa de absenteísmo e pelo valor monetário gerado em cada especialidade.

As taxas de absenteísmo anuais para consultas e exames especializados foram calculadas com a divisão do total de procedimentos não confirmados (absenteísmo) (numerador) pelo total de procedimentos agendados (denominador) para cada especialidade, multiplicando-se o resultado por cem.

Os valores monetários estimados para desperdício foram obtidos mediante a multiplicação do total de procedimentos não confirmados (absenteísmo) anualmente por especialidade pelos valores atribuídos a cada procedimento segundo a natureza administrativa do local executante.

Para as análises foi utilizado o Programa Microsoft Office Excel ${ }^{\circledR}$. O estudo foi autorizado pela Sesa-ES por meio do Termo de Anuência, datado de 26 de setembro de 2017, constante no processo $\mathrm{n}^{\circ} 79619819 / 2017$, e aprovado pelo Comitê de Ética em Pesquisa da Universidade Federal do Espírito Santo (Ufes), respeitando a resolução do Conselho Nacional de Saúde, aprovado em 02 de maio de 2018, protocolo $\mathrm{n}^{\mathrm{0}} 2.631 .695$. 


\section{Resultados}

Durante o período estudado, foram contabilizados 666.182 procedimentos de consultas e 336.537 exames especializados, em um total de 1.002.719 procedimentos, o que corresponde a $\mathrm{R} \$$ 18.566.462,03 de valores monetários desperdiçados atribuídos ao absenteísmo. Deve-se destacar que esses valores devem ser relativizados, visto que a pesquisa apresentou limitações devido às fragilidades do banco de dados gerado no SisReg-ES e à falta de análise econômica por local executante não oferecendo informações sobre o custo real do procedimento.

As consultas especializadas, agendadas para 34 especialidades, apresentaram taxa média de absenteísmo no período de 38,6\% (257.025 consultas) e um desperdício associado ao absenteísmo estimado de $\mathrm{R} \$ 3.558 .837,88$. A especialidade, com todas as consultas clínicas somadas, que apresentou o menor valor monetário com o absenteísmo nos três anos de estudo foi a de médico oncologista clínico ( $\mathrm{R} \$ 880,00)$, e a que apresentou o maior valor foi de médico oftalmologista ( $\mathrm{R} \$ 558.262,20)$. Os índices de absenteísmo por especialidades variaram de $0 \%$ (cirurgião-dentista para pacientes com necessidades especiais) a $75,9 \%$ (fisioterapeuta geral). Destaque-se que as consultas agendadas com cirurgião-dentista para pacientes com necessidades especiais não foram oferecidas nos anos de 2015 e 2016 (tabela 1).
Apenas para a especialidade médica cancerologista cirúrgico $(2,9 \%)$ a taxa de absenteísmo foi inferior a $30 \%(26,4 \%)$, com um desperdício atribuído ao absenteísmo de R\$900,00. Entre o total, 19 especialidades, o equivalente a $55,9 \%$, tiveram uma variação da taxa de absenteísmo entre $30 \%$ e $40 \%$, sendo as consultas com cirurgião-dentista bucomaxilofacial (30,2\%) as que apresentaram a menor taxa do grupo, e as consultas com médico pneumologista as que apresentaram a maior taxa (39,4\%). Esse grupo totalizou um desperdício estimado em R\$ 1.761.422,86 pelo absenteísmo. Nove especialidades (26,5\%) apresentaram uma variação da taxa de absenteísmo entre $40 \%$ e $50 \%$, tendo as consultas com médicos gastroenterologista e neurologista menor taxa $(40,1 \%)$, e as consultas com médico geneticista a maior taxa $(47,4 \%)$. Essas especialidades somadas apresentaram um desperdício de $\mathrm{R} \$ 1.671 .403,22$.

Duas especialidades (5,9\%) apresentaram a taxa de absenteísmo entre $50 \%$ e inferior a $60 \%$ - médico cirurgião pediátrico $(52,5 \%)$ e médico infectologista (58,3\%), com um desperdício estimado de $\mathrm{R} \$ 62.831,80$. Somente as consultas com médico fisiatra (2,9\%) apresentaram taxa de absenteísmo superior a $60 \%$ (68,4\%), com desperdício estimado de $\mathrm{R} \$ 45.630,00$. Para as consultas de fisioterapeuta geral (2,9\%), foram encontradas as maiores taxas de absenteísmo (75,9\%), com desperdício estimado em $\mathrm{R} \$ 16.650,00$.

Tabela 1. Descrição do absenteísmo em consultas especializadas na Região de Saúde Metropolitana, Espírito Santo, Brasil. 2014-2016

\begin{tabular}{|c|c|c|c|c|c|c|c|c|c|c|c|c|c|c|}
\hline \multicolumn{4}{|c|}{2014} & \multicolumn{4}{|c|}{2015} & \multicolumn{3}{|c|}{2016} & \multicolumn{2}{|r|}{ 2014-2016 } & \multicolumn{2}{|c|}{ Derperdício } \\
\hline A & C & Abs. & Valor R\$ & A & C & Abs. & Valor R\$ & A & C & Abs. & Valor R\$ & Ag. Total & $\begin{array}{r}\text { Tx. Abs. } \\
\text { N (\%) }\end{array}$ & Valor $(R \$)$ \\
\hline \multicolumn{15}{|c|}{ Médico Oncologista Clínico } \\
\hline 104 & 73 & 31 & 310,00 & 73 & 50 & 23 & 230,00 & 88 & 54 & 34 & 340,00 & 265 & $88(33,2)$ & 880,00 \\
\hline \multicolumn{15}{|c|}{ Médico Cancerologista Cirúrgico } \\
\hline 94 & 73 & 21 & 210,00 & 109 & 81 & 28 & 280,00 & 127 & 89 & 38 & 410,00 & 330 & $87(26,4)$ & 900,00 \\
\hline \multicolumn{15}{|c|}{ Cirurgião-Dentista - Clínico Geral } \\
\hline 67 & 38 & 29 & 390,00 & 106 & 59 & 47 & 760,00 & 109 & 84 & 25 & 390,00 & 282 & $101(35,8)$ & $1.540,00$ \\
\hline \multicolumn{15}{|c|}{ Médico Geneticista } \\
\hline 34 & 15 & 19 & 380,00 & 54 & 28 & 26 & 520,00 & 102 & 57 & 45 & 900,00 & 190 & $90(47,4)$ & $1.800,00$ \\
\hline
\end{tabular}


Tabela 1. (cont.)

\begin{tabular}{|c|c|c|c|c|c|c|c|c|c|c|c|c|c|c|}
\hline \multicolumn{4}{|c|}{2014} & \multicolumn{4}{|c|}{2015} & \multicolumn{3}{|c|}{2016} & \multicolumn{2}{|r|}{ 2014-2016 } & \multicolumn{2}{|c|}{ Derperdício } \\
\hline A & C & Abs. & Valor R\$ & A & C & Abs. & Valor R\$ & A & C & Abs. & Valor R\$ & Ag. Total & $\begin{array}{r}\text { Tx. Abs. } \\
\text { N (\%) }\end{array}$ & Valor $(R \$)$ \\
\hline \multicolumn{15}{|c|}{ Cirurgião-Dentista Bucomaxilofacial } \\
\hline 90 & 61 & 29 & 290,00 & 186 & 124 & 62 & $3.037,75$ & 254 & 185 & 69 & $3.204,46$ & 530 & $160(30,2)$ & $6.532,21$ \\
\hline \multicolumn{15}{|c|}{ Médico Cirurgião Torácico } \\
\hline 36 & 22 & 14 & 140,00 & 96 & 59 & 37 & 790,13 & 175 & 107 & 68 & $5.728,92$ & 307 & $119(38,8)$ & $6.659,05$ \\
\hline \multicolumn{15}{|c|}{ Médico Cirurgião Plástico } \\
\hline 879 & 511 & 368 & $4.550,00$ & 1.216 & 776 & 440 & $5.050,00$ & 1.049 & 653 & 396 & $4.620,00$ & 3144 & $1.204(38,3)$ & $14.220,00$ \\
\hline \multicolumn{15}{|c|}{ Médico Cirurgião de Cabeça e Pescoço } \\
\hline 457 & 310 & 147 & $10.307,00$ & 542 & 347 & 195 & $2.220,00$ & 969 & 646 & 323 & $3.480,00$ & 1968 & $665(33,8)$ & $16.007,00$ \\
\hline \multicolumn{15}{|c|}{ Fisioterapeuta Geral } \\
\hline 908 & 243 & 665 & $6.650,00$ & 714 & 91 & 623 & $6.230,00$ & 572 & 195 & 377 & $3.770,00$ & 2194 & $1.665(75,9)$ & $16.650,00$ \\
\hline \multicolumn{15}{|c|}{ Médico Mastologista } \\
\hline 2.137 & 1.493 & 644 & $6.560,00$ & 2.065 & 1.427 & 638 & $7.280,00$ & 1.943 & 1.334 & 609 & $6.620,00$ & 6145 & $1.891(30,8)$ & $20.460,00$ \\
\hline \multicolumn{15}{|c|}{ Médico Infectologista } \\
\hline 425 & 259 & 166 & $9.821,80$ & 305 & 147 & 158 & $1.950,00$ & 924 & 283 & 641 & $11.810,00$ & 1654 & $965(58,3)$ & $23.581,80$ \\
\hline \multicolumn{15}{|c|}{ Médico Geriatra } \\
\hline 2.574 & 1.796 & 778 & $7.780,00$ & 2.403 & 1.586 & 817 & $8.170,00$ & 2.466 & 1.618 & 848 & $8.480,00$ & 7443 & $2.443(32,8)$ & $24.430,00$ \\
\hline \multicolumn{15}{|c|}{ Médico Hematologista } \\
\hline 3.102 & 2.025 & 1.077 & $11.370,00$ & 3.610 & 2.243 & 1.367 & $14.870,00$ & 3.229 & 2.065 & 1.164 & $12.920,00$ & 9941 & $3.608(36,3)$ & $39.160,00$ \\
\hline \multicolumn{15}{|c|}{ Médico Cirurgião Pediátrico } \\
\hline 1.497 & 594 & 903 & $9.720,00$ & 2.030 & 1.041 & 989 & $11.520,00$ & 2.824 & 1.380 & 1.444 & $18.010,00$ & 6351 & $3.336(52,5)$ & $39.250,00$ \\
\hline \multicolumn{15}{|c|}{ Médico Ginecologista e Obstetra } \\
\hline 2.897 & 2.000 & 897 & $10.830,00$ & 3.185 & 2.185 & 1.000 & $11.040,00$ & 3.978 & 2.389 & 1.589 & $17.450,00$ & 10060 & $3.486(34,7)$ & $39.320,00$ \\
\hline \multicolumn{15}{|c|}{ Médico Fisiatra } \\
\hline 1.855 & 6.38 & 1.217 & $12.170,00$ & 2.322 & 635 & 1.687 & $16.870,00$ & 2.493 & 834 & 1.659 & $16.590,00$ & 6670 & $4.563(68,4)$ & $45.630,00$ \\
\hline \multicolumn{15}{|c|}{ Médico Nefrologista } \\
\hline 6.226 & 4.229 & 1.997 & $20.120,00$ & 4.152 & 2.738 & 1.414 & $14.980,00$ & 2.895 & 1.856 & 1.039 & $10.590,00$ & 13273 & $4.450(33,5)$ & $45.690,00$ \\
\hline \multicolumn{15}{|c|}{ Médico Alergista e Imunologista } \\
\hline 3.369 & 2.117 & 1.252 & $12.520,00$ & 4.845 & 2.956 & 1.889 & $18.890,00$ & 4.959 & 3.062 & 1.897 & $18.970,00$ & 13173 & $5.038(38,2)$ & $50.380,00$ \\
\hline \multicolumn{15}{|c|}{ Médico Neurocirurgião } \\
\hline 1.157 & 830 & 327 & $39.689,80$ & 572 & 352 & 220 & $8.481,00$ & 491 & 245 & 246 & $2.600,00$ & 2220 & $793(35,7)$ & $50.770,80$ \\
\hline \multicolumn{15}{|c|}{ Médico Pneumologista } \\
\hline 4.594 & 2.838 & 1.756 & $21.505,00$ & 4.460 & 2.763 & 1.697 & $20.545,00$ & 2.535 & 1.424 & 1.111 & $11.350,00$ & 11589 & $4.564(39,4)$ & $53.400,00$ \\
\hline \multicolumn{15}{|c|}{ Médico Reumatologista } \\
\hline 5.494 & 4.082 & 1.412 & $14.230,00$ & 7.851 & 5.140 & 2.711 & $28.100,00$ & 6.778 & 4.665 & 2.113 & $21.710,00$ & 20123 & $6.236(31,0)$ & $64.040,00$ \\
\hline \multicolumn{15}{|c|}{ Médico Coloproctologista } \\
\hline 3.147 & 1.889 & 1.258 & $14.270,00$ & 3.754 & 2.028 & 1.726 & $19.190,00$ & 6.264 & 3.169 & 3.095 & $33.890,00$ & 13165 & $6.079(46,2)$ & $67.350,00$ \\
\hline
\end{tabular}


Tabela 1. (cont.)

\begin{tabular}{|c|c|c|c|c|c|c|c|c|c|c|c|c|c|c|}
\hline \multicolumn{4}{|c|}{2014} & \multicolumn{4}{|c|}{2015} & \multicolumn{3}{|c|}{2016} & \multicolumn{2}{|r|}{ 2014-2016 } & \multicolumn{2}{|c|}{ Derperdício } \\
\hline A & C & Abs. & Valor R\$ & A & C & Abs. & Valor $\mathbf{R} \$$ & A & C & Abs. & Valor R\$ & Ag. Total & $\begin{array}{r}\text { Tx. Abs. } \\
\text { N (\%) }\end{array}$ & Valor (R\$) \\
\hline \multicolumn{15}{|c|}{ Médico Cardiologista } \\
\hline 7.369 & 4.803 & 2.566 & $52.759,60$ & 6.317 & 3.724 & 2.593 & $29.000,00$ & 10.013 & 5.598 & 4.415 & $45.470,00$ & 23699 & $9.574(40,4)$ & $127.229,60$ \\
\hline \multicolumn{15}{|c|}{ Médico Angiologista } \\
\hline 2.559 & 1.425 & 1.134 & $65.765,80$ & 5.957 & 3.570 & 2.387 & $43.046,95$ & 7.448 & 4.160 & 3.288 & $42.297,00$ & 15964 & $6.809(42,7)$ & $151.109,75$ \\
\hline \multicolumn{15}{|c|}{ Médico Cirurgião Geral } \\
\hline 7.019 & 4.219 & 2.800 & $93.084,20$ & 6.810 & 4.050 & 2.760 & $38.936,60$ & 4.234 & 2.493 & 1.741 & $20.720,00$ & 18063 & $7.301(40,4)$ & $152.740,80$ \\
\hline \multicolumn{15}{|c|}{ Médico Psiquiatra } \\
\hline 13.396 & 8.268 & 5.128 & $53.956,00$ & 15.373 & 9.323 & 6.050 & $60.500,00$ & 12.296 & 7.736 & 4.560 & $45.600,00$ & 41065 & $15.738(38,3)$ & $160.056,00$ \\
\hline \multicolumn{15}{|c|}{ Médico Neurologista } \\
\hline 8.654 & 5.260 & 3.394 & $58.533,54$ & 10.915 & 6.598 & 4.317 & $64.084,45$ & 7.683 & 4.465 & 3.218 & $40.185,13$ & 27252 & $10.929(40,1)$ & $162.803,12$ \\
\hline \multicolumn{15}{|c|}{ Médico Urologista } \\
\hline 14.736 & 10.237 & 4.499 & $79.112,00$ & 15.638 & 11.052 & 4.586 & $49.660,80$ & 14.798 & 9.522 & 5.276 & $55.860,00$ & 45172 & $14.361(31,8)$ & $184.632,80$ \\
\hline \multicolumn{15}{|c|}{ Médico Gastroenterologista } \\
\hline 8.088 & 5.382 & 2.706 & $84.532,60$ & 6.571 & 3.930 & 2.641 & $54.047,20$ & 11.472 & 6.340 & 5.132 & $52.840,00$ & 26131 & $10.479(40,1)$ & $191.419,80$ \\
\hline \multicolumn{15}{|c|}{ Médico Endocrinologista e Metabologista } \\
\hline 16.217 & 10.945 & 5.272 & $56.635,00$ & 18.678 & 12.006 & 6.672 & $69.090,00$ & 19.263 & 12.731 & 6.532 & $68.400,00$ & 54158 & $18.476(34,1)$ & $194.125,00$ \\
\hline \multicolumn{15}{|c|}{ Médico Otorrinolaringologista } \\
\hline 18.850 & 11.700 & 7.150 & $83.404,40$ & 21.081 & 13.261 & 7.820 & $84.963,40$ & 15.917 & 9.792 & 6.125 & $62.490,00$ & 55848 & $21.095(37,8)$ & $230.857,80$ \\
\hline \multicolumn{15}{|c|}{ Médico Dermatologista } \\
\hline 23.810 & 13.602 & 10.208 & $104.810,00$ & 25.478 & 14.784 & 10.694 & $111.200,00$ & 26.782 & 16.106 & 10.676 & $110.600,00$ & 76070 & $3.1578(41,5)$ & $326.610,00$ \\
\hline \multicolumn{15}{|c|}{ Médico Ortopedista } \\
\hline 20.425 & 12.189 & 8.236 & $185.651,44$ & 28.408 & 16.830 & 11.578 & $123.410,37$ & 41.935 & 24.662 & 17.273 & $181.278,34$ & 90768 & $37.087(40,9)$ & $490.340,15$ \\
\hline \multicolumn{15}{|c|}{ Médico Oftalmologista } \\
\hline 18.562 & 12.536 & 6.026 & $131.920,00$ & 19.942 & 12.342 & 7.600 & $204.292,68$ & 22.468 & 14.127 & 8.341 & $222.049,52$ & 60972 & $21.967(36,0)$ & $558.262,20$ \\
\hline
\end{tabular}

Cirurgião-Dentista - Paciente Com Necessidade Especial

\begin{tabular}{rrrrrrrrrrrrrrr}
3 & 3 & 0 & - & 0 & 0 & 0 & - & 0 & 0 & 0 & - & 3 & - \\
\hline \multicolumn{10}{c}{ Total Geral } \\
200.831 & 126.705 & 74.126 & $1.263 .978,18$ & 225.818 & 138.326 & 87.492 & $1.133 .236,33$ & 239.533 & 144.126 & 95407 & $1.161 .623,37$ & 666.182 & 257.025 & $3.558 .837,88$ \\
\hline
\end{tabular}

Fonte: Elaboração própria a partir de dados do SisReg/Sesa-ES

A - agendada; C - confirmada; Abs. - absenteísmo; Ag. Total - agendada total; N - absenteísmo total; Tx. Abs. - Taxa de absenteísmo.

O total de consultas especializadas agendadas no período cresceu de 200.831, em 2014, para 239.533, em 2016 (aumento de 19,3\%) (gráfico 1), destacando-se os crescimentos absolutos nas especialidades de ortopedia e oftalmologia (tabela 1).
O absenteísmo em consultas especializadas teve aumento médio de $2,9 \%$ entre 2014 e 2016. Para as consultas especializadas, no ano de 2014, em um total de 200.831 agendadas, a taxa de absenteísmo foi de $36,9 \%$, o equivalente a 74.126 consultas em 2015 , em 
um total de 225.818; a taxa de absenteísmo foi de $38,7 \%$ - 87.492 consultas -, e, em 2016, em um total de 239.533, a taxa de absenteísmo foi de $39,8 \%$ - 95.407 consultas -, sendo a taxa média de absenteísmo para os anos estudados de $38,6 \%$, em um total de 257.025 consultas não realizadas (gráfico 1).

A taxa média de absenteísmo para os exames especializados foi de $32,1 \%$, o equivalente a
108.103 exames perdidos. No período estudado, foram 108.103 exames agendados e não realizados, com um desperdício estimado em R\$ 15.007.624,15 (tabela 2). Para exames especializados, a taxa de absenteísmo vem decrescendo anualmente, sendo com 34,3\% em 2014, 32,7\% em 2015 e 29,7\% em 2016. Em contraste, a oferta cresceu $9,7 \%$ entre 2014 e 2015, e 13,1\% entre 2015 e 2016 (gráfico 1).

Gráfico 1. Taxas de absenteísmo e oferta de exames e consultas especializadas na Região de Saúde Metropolitana, Espírito Santo, Brasil. 2014-2016

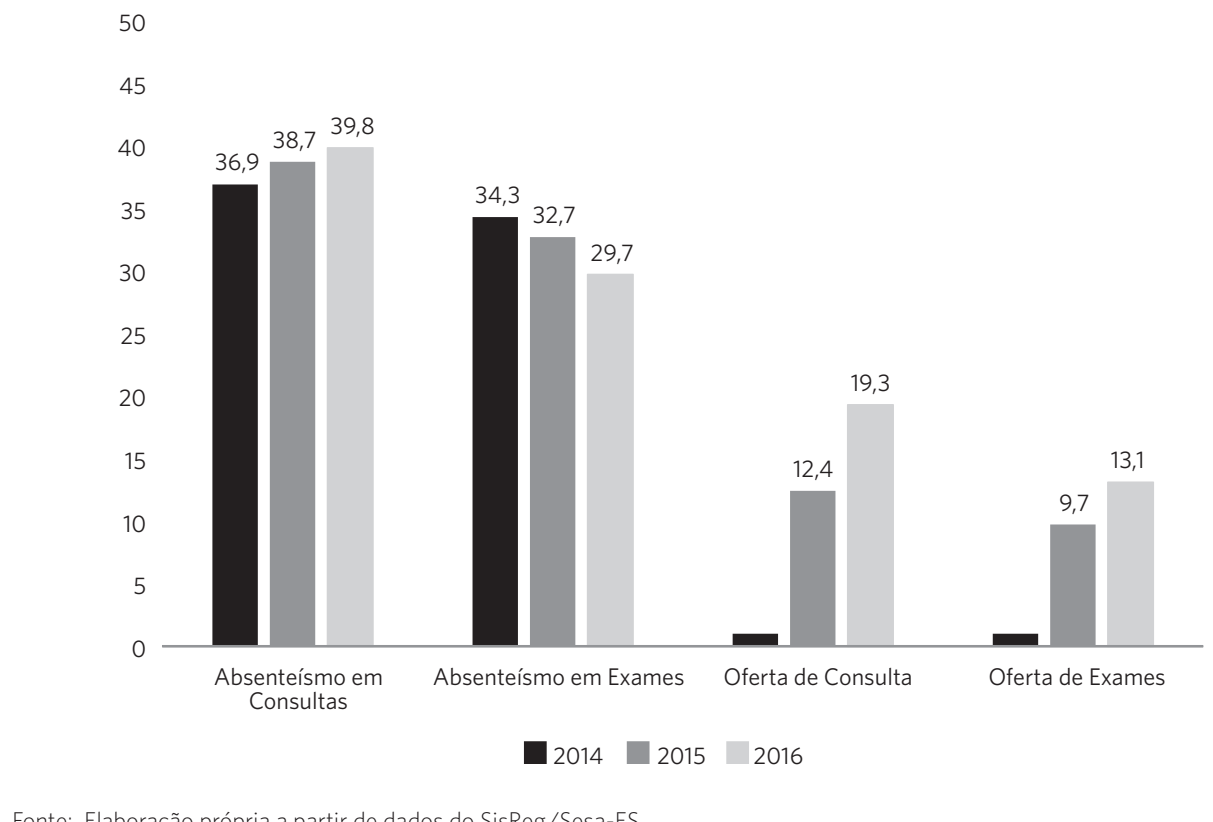

Fonte: Elaboração própria a partir de dados do SisReg/Sesa-ES.

Durante o período analisado, foram agendados 336.537 exames especializados, e os índices de absenteísmo variaram de 22,3\% (diagnóstico por tomografia) a 100\% (terapias especializadas), relacionados com os exames de litotripsia extracorpórea. Dos procedimentos com finalidade diagnóstica (grupo 02), quatro exames apresentaram absenteísmo entre 20,0\% e 30,0\%. Os exames do subgrupo 02.06, diagnóstico por tomografia, apresentaram as menores taxas de absenteísmo (22,3\%); e os do subgrupo 02.01 , exame com coleta de material, as maiores taxas $(28,2 \%)$. Os outros quatro exames especializados nesse grupo tiveram taxas de absenteísmo entre 30,0\% e 40,0\%, sendo os exames do subgrupo 02.09, diagnóstico por endoscopia, a menor taxa (30,4\%); e os do subgrupo 02.04, diagnóstico por radiologia, a maior taxa (39,1\%). Os exames do grupo 02 somados representaram um desperdício estimado de $\mathrm{R} \$ 13.774 .088,92$, o que corresponde a $91,8 \%$ do total de desperdício com o absenteísmo em exames especializados.

Procedimentos vinculados ao grupo 04 procedimentos cirúrgicos - tiveram taxas de absenteísmo entre $30,0 \%$ e 70,0\%. Cirurgia 
do aparelho de visão (subgrupo 04.05) apresentou a menor taxa de absenteísmo (34,5\%); e o subgrupo 04.04 - cirurgia de vias aéreas superiores, cabeça e pescoço - teve a maior taxa de absenteísmo (66,7\%). Os valores dos procedimentos do grupo 03 e 04 somados representaram um desperdício de $\mathrm{R} \$ 1.233 .535,23$ (8,2\% do total de desperdício monetário acumulado nos grupos 02, 03 e 04 dos exames especializados), conforme tabela 2.

Tabela 2. Descrição do absenteísmo em exames especializados na Região de Saúde Metropolitana, Espírito Santo, Brasil. 2014-2016

\begin{tabular}{|c|c|c|c|c|c|c|c|c|c|c|c|c|c|c|}
\hline \multicolumn{4}{|c|}{2014} & \multicolumn{4}{|c|}{2015} & \multicolumn{3}{|c|}{2016} & \multicolumn{2}{|r|}{ 2014-2016 } & \multicolumn{2}{|c|}{ Desperdício } \\
\hline A & C & Abs. & Valor R\$ & A & C & Abs. & Valor R\$ & A & C & Abs. & Valor R\$ & Ag. Total & $\begin{array}{r}\text { Tx. Abs. } \\
\text { N (\%) }\end{array}$ & Valor $(R \$)$ \\
\hline \multicolumn{15}{|c|}{ 02-PROCEDIMENTOS COM FINALIDADE DIAGNÓSTICA } \\
\hline \multicolumn{15}{|c|}{..02.01-Coleta de material } \\
\hline 1.354 & 927 & 427 & $142.603,39$ & 1.330 & 979 & 351 & $121.878,46$ & 1.391 & 1.018 & 373 & $123.153,30$ & 4.075 & $1.151(28,2)$ & $387.635,15$ \\
\hline \multicolumn{15}{|c|}{.02.06-Diagnóstico por tomografia } \\
\hline 6.754 & 5.139 & 1.615 & $226.876,80$ & 7.987 & 6.179 & 1.808 & $266.716,69$ & 9.308 & 7.365 & 1.943 & $274.201,81$ & 24.049 & $5.366(22,3)$ & $767.795,30$ \\
\hline \multicolumn{15}{|c|}{..02.08-Diagnóstico por medicina nuclear in vivo } \\
\hline 4.419 & 3.211 & 1.208 & $370.829,93$ & 3.599 & 2.825 & 774 & $254.287,46$ & 3.499 & 2.631 & 868 & $258.294,61$ & 11.517 & $2.850(24,7)$ & $883.412,00$ \\
\hline \multicolumn{15}{|c|}{.02.11-Métodos diagnósticos em especialidades } \\
\hline 14.176 & 9.990 & 4.186 & $227.820,06$ & 16.531 & 11.654 & 4.877 & $310.527,67$ & 16.664 & 11.192 & 5.472 & $424.720,26$ & 47.371 & $14.535(30,7)$ & $963.067,99$ \\
\hline \multicolumn{15}{|c|}{.02.04-Diagnóstico por radiologia } \\
\hline 27.771 & 15.325 & 12.446 & $432.363,23$ & 26.833 & 16.408 & 10.425 & $427.419,72$ & 23.274 & 15.692 & 7.582 & $363.127,38$ & 77.878 & $30.453(39,1)$ & $1.222 .910,33$ \\
\hline \multicolumn{15}{|c|}{..02.07-Diagnóstico por ressonância magnética } \\
\hline 7.191 & 5.452 & 1.739 & $544.493,45$ & 8.710 & 6.881 & 1.829 & $573.885,99$ & 9.814 & 7.565 & 2.249 & $766.104,75$ & 25.715 & $5.817(22,7)$ & $1.884 .484,19$ \\
\hline \multicolumn{15}{|c|}{..02.05-Diagnóstico por ultrassonografia } \\
\hline 28.601 & 18.511 & 10.090 & $989.998,75$ & 30.988 & 19.542 & 11.446 & 1.097.388,01 & 32.368 & 22.578 & 9.790 & $927.641,32$ & 91.957 & $31.326(34,1)$ & $3.015 .028,08$ \\
\hline \multicolumn{15}{|c|}{.02.09-Diagnóstico por endoscopia } \\
\hline 12.992 & 9.223 & 3.769 & $1.008 .499,89$ & 16.905 & 11.611 & 5.294 & $1.719 .830,13$ & 19.567 & 13.592 & 5.975 & $1.921 .425,86$ & 49.464 & $15.038(30,4)$ & $4.649 .755,88$ \\
\hline
\end{tabular}

Total Procedimentos com Finalidade Diagnóstica

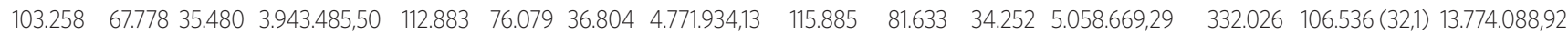
03-PROCEDIMENTOS CLÍNICOS

.03.09-Terapias especializadas

\begin{tabular}{|c|c|c|c|c|c|c|c|c|c|c|c|c|c|c|}
\hline 1 & - & 1 & 172,00 & - & - & - & - & - & - & - & - & 1 & $1(100,0)$ & 172,00 \\
\hline \multicolumn{15}{|c|}{ Total Procedimentos com Terapias Especializadas } \\
\hline 1 & - & 1 & 172,00 & - & - & - & - & - & - & - & - & 1 & $1(100,0)$ & 172,00 \\
\hline \multicolumn{15}{|c|}{ 04-PROCEDIMENTOS CIRÚRGICOS } \\
\hline \multicolumn{15}{|c|}{..04.04-Cirurgia vias aéreas super, cabeça pescoço } \\
\hline 2 & 1 & 1 & 48,42 & - & - & - & - & 1 & - & 1 & 48,42 & 3 & $2(66,7)$ & 96,84 \\
\hline
\end{tabular}

.04.06-Cirurgia do aparelho Circulatório

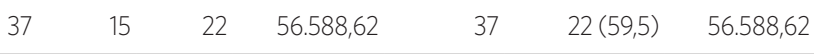




\begin{tabular}{|c|c|c|c|c|c|c|c|c|c|c|c|c|c|c|}
\hline \multicolumn{4}{|c|}{2014} & \multicolumn{4}{|c|}{2015} & \multicolumn{4}{|c|}{2016} & $2014-2016$ & \multicolumn{2}{|c|}{ Desperdício } \\
\hline A & C & Abs. & Valor R\$ & A & $\mathrm{C}$ & Abs. & Valor $\mathbf{R} \$$ & A & $\mathrm{C}$ & Abs. & Valor R\$ & Ag. Total & $\begin{array}{r}\text { Tx. Abs. } \\
\text { N (\%) }\end{array}$ & Valor (R\$) \\
\hline \multicolumn{15}{|c|}{ 04-PROCEDIMENTOS CIRÚRGICOS } \\
\hline \multicolumn{15}{|c|}{.04.05-Cirurgia do aparelho da visão } \\
\hline 991 & 700 & 291 & $213.393,62$ & 1.476 & 916 & 560 & $452.180,71$ & 2.003 & 1.312 & 691 & $511.103,44$ & 4.470 & $1.542(34,5)$ & $1.176 .677,77$ \\
\hline \multicolumn{15}{|c|}{ Total Procedimentos Cirúrgicos } \\
\hline 993 & 701 & 292 & $213.442,04$ & 1.476 & 916 & 560 & $452.180,71$ & 2.041 & 1.327 & 714 & $567.740,48$ & 4.510 & $1.566(34,7)$ & $1.233 .363,23$ \\
\hline \multicolumn{15}{|c|}{ Total Geral } \\
\hline 104.252 & 68.479 & 35.773 & $4.157 .099,54$ & 114.359 & 76.995 & 37.364 & $5.224 .114,84$ & 117.926 & 82.960 & 34.966 & $5.626 .409,77$ & 336.537 & $108.103(32,1)$ & $15.007 .624,15$ \\
\hline
\end{tabular}

\section{Discussão}

Os estudos publicados sobre absenteísmo, tanto no Brasil como no exterior, são enfáticos em afirmar que o ele causa prejuízos monetários, mas poucos apresentam uma abordagem fundamentada em avaliação econômica e métodos analíticos dos serviços para estimar valores envolvidos 4 . O presente estudo, portanto, é pioneiro ao atribuir valores monetários ao não comparecimento dos usuários do SUS a procedimentos agendados no estado do ES.

A estimativa de desperdício monetário acumulado nos três anos estudados com o absenteísmo dos usuários em consultas, exames e outros procedimentos especializados na RSM-ES foi de R $\$ 18.566 .462,03$, com valores atualizados das tabelas utilizadas, para o ano de 2018. Os resultados corroboram os prejuízos monetários gerados pelo absenteísmo, ainda que as estimativas sejam limitadas pelos dados disponíveis em função das estruturas administrativas existentes nas diversas modalidades de gestão, principalmente nos serviços públicos, deixando a análise muito restrita a valores da Tabela SUS.

Houve crescimento tanto da oferta de consultas e exames como da taxa de absenteísmo em consultas, o que fundamenta a preocupação da gestão estadual com o tema ${ }^{28}$. Os resultados mostram que as taxas de absenteísmo em consultas especializadas $(38,6 \%)$ e em exames especializados (32,1\%) na RSM-ES são altas e estão compatíveis com outros estudos realizados no Brasil que pesquisaram a atenção especializada, confirmando que as taxas altas não são exclusivas daqui, destacando em São Paulo (34,4\%) ${ }^{\mathbf{9}}$, em Florianópolis $(34,4 \%)^{2}$ e em João Pessoa $(39,8 \%)^{3}$, preocupando aos gestores, evidenciando que os esforços somados para ampliar a oferta acabam não se efetivando.

A especialidade odontológica para pacientes com necessidades especiais teve taxa zero de absenteísmo, o que, segundo a gerência da Central de Regulação, pode ser justificado pela especialidade ter sido ofertada somente em 2014 e pela possibilidade de baixa oferta de vagas gerar uma grande expectativa nos familiares. Os pacientes especiais de acordo com os comprometimentos não são atendidos na atenção básica odontológica e são encaminhados para a atenção especializada. A especialidade médico cancerologista cirúrgico apresentou a menor taxa de absenteísmo $(26,4 \%)$ e pode ser justificada pela gravidade da patologia já diagnosticada e pela necessidade de rápida intervenção.

A consulta de fisioterapeuta geral apresentou a maior taxa $(75,8 \%)$, o que está em acordo com a revisão sistemática de Dantas et 
al. ${ }^{7}$, que encontraram a taxa de $79,2 \%$, sendo as causas mais frequentes as limitações de locomoção devido aos agravos das patologias crônicas, estando em sintonia com o relato da gerência da Central de Regulação, segundo o qual as faltas podem ser justificadas por falha no registro de comparecimento, por agravos da patologia e também, para alguns casos, a melhora dos sintomas.

Procedimentos com finalidade diagnóstica, como tomografia e ressonância magnética, tiveram as menores taxas de absenteísmo $22,3 \%$ e $22,7 \%$ respectivamente. O menor absenteísmo do grupo pode ser explicado pelo fato de esses exames terem maior complexidade tecnológica e serem, em tese, solicitados nos casos de uma clínica mais definida, que requer um exame mais qualificado para auxiliar no diagnóstico e conduta. Apesar de serem menores, essas taxas de absenteísmo são significativas, e os valores estimados para desperdício são altos, por serem exames de alta densidade tecnológica, acarretando um desperdício de $\mathrm{R} \$ 2.652 .279,49$, o equivalente a 19,3\% do total monetário desperdiçado no grupo 02. Nesse mesmo grupo, observou-se que os exames com menor densidade tecnológica, subgrupo 04, diagnóstico por radiologia, geralmente solicitados quando a clínica não está muito definida, são os que apresentam as maiores taxas de absenteísmo (39,1\%). As taxas de absenteísmo altas podem ser explicadas por diversos motivos, podendo-se destacar o longo tempo de espera, que pode cursar com melhora ou a piora dos sintomas, podendo o usuário buscar serviços de emergência ou pagar na rede privada ${ }^{3,9}$.

O aumento da oferta de consultas nos 20 municípios da RSM-ES no período de estudo evidencia os esforços da gestão para implementar o Plano de Intervenção Regional (PIR), baseado no Plano Diretor de Regionalização (PDR) do ES de 2011, que dividiu o estado em quatro regiões de saúde. O PIR foi influenciado pelo Decreto Federal no 7.508, de 28 de junho de 2011, que regulamentou a Lei Federal $\mathrm{n}^{\mathrm{o}} 8.080 / 90$ e reafirmou a necessidade da reorganização das redes de atenção no âmbito das regiões de saúde ${ }^{\mathbf{1 9}, 33}$.

A regionalização, entendida aqui como a diretriz organizativa do SUS, que orienta os processos de descentralização das ações e dos serviços de saúde e a pactuação entre os gestores nos níveis federal, estadual e municipal, visa organizar redes de atenção com distintas densidades tecnológicas ${ }^{34}$. Ela orientou a PPI entre o Estado e municípios na região de saúde, contemplando os desafios da integralidade do cuidado e das respostas às necessidades da população de disponibilizar serviços de saúde mais próximos do usuário, principalmente na atenção especializada e na alta complexidade ${ }^{33}$.

Considera-se aqui o conceito de integralidade defendido por Giovanella et al. ${ }^{35}$, isto é, a garantia da assistência nos três níveis de atenção à saúde e a articulação das ações de promoção, prevenção e recuperação, em sintonia com o conceito defendido por Pinheiro ${ }^{36}$. Para esta última autora, integralidade é uma construção coletiva que ganha forma e expressão no espaço do encontro dos diferentes sujeitos implicados na produção do cuidado e em defesa da vida.

A atenção especializada é considerada um anteparo para o bom desempenho e avanço na qualidade do sistema de saúde, podendo constituir-se em um nó crítico dos mais complexos. Por isso, tem sido destacada por gestores e pesquisadores como um desafio para atingir a integralidade como diretriz do SUS 23,37. Segundo Mendes ${ }^{\mathbf{3 8}}$, para alcançar a integralidade, há a necessidade de vincular a assistência e a integração dos serviços por meio de redes de saúde, regionalizadas e hierarquizada, considerando-se a dificuldade em dispor da totalidade de recursos e competências para a solução de todos os problemas de saúde de uma população. No período do estudo, as redes no ES ainda não estavam definidas. A implantação iniciou-se em setembro de 2017 na região norte do estado, sendo três implantadas em 2018.

Para organizar o fluxo nos três níveis de atenção, a regulação do acesso é considerada 
um valioso instrumento da gestão; a qual busca estabelecer um equilíbrio entre a demanda, a oferta e o financiamento ${ }^{27}$. As taxas de absenteísmo crescentes na RSM-ES indicam que, apesar de a oferta das vagas reguladas ter aumentado, o absenteísmo contribuiu, na prática, para reduzir o acesso à atenção especializada, evidenciando oportunidades de assistência perdidas e contribuindo para aumentar a fila de espera por procedimentos ${ }^{9}$.

$\mathrm{O}$ acesso à média complexidade é entendido aqui como a oportunidade de buscar e obter serviços de saúde adequados às necessidades do usuário ${ }^{38}$. A ampliação do acesso às ações e aos serviços é um dos grandes desafios do SUS e exige dos gestores a organização e mecanismos que visem consolidar o sistema ${ }^{23}$. É possível compreender que acesso vai além da utilização do sistema de saúde. É um conceito de múltiplas dimensões, composto por fatores financeiros e não financeiros, que pode variar ao longo do tempo com a evolução e o surgimento de novas necessidades para a sociedade ${ }^{39}$.

Os desafios para a oferta da atenção especializada na RSM-ES são grandes, considerando suas características. A realidade dos 20 municípios é muito diferente. Em oito deles, a população de residentes está em torno de 15 mil habitantes, e em quatro, ultrapassa $300 \mathrm{mil}$ habitantes, gerando assim grandes diferenças em relação aos investimentos na atenção à saúde. Alguns municípios dispõem somente de serviços básicos evidenciando a necessidade de organização da atenção especializada nas redes de saúde, entre os sistemas municipal e estadual, no âmbito da região ${ }^{33}$. A atenção especializada é estratégica e necessária para dar efetividade e sequência à atenção básica, complementando-a e provendo assistência especializada a quem dela necessitar ${ }^{40}$. Assim, o absenteísmo na atenção especializada é uma preocupação constante para os gestores tanto pela desassistência quanto pelo desperdício ${ }^{2,9}$.

Os custos da atenção especializada são altos e englobam procedimentos de maior densidade tecnológica, as chamadas tecnologias especializadas $^{41}$. Entre as pesquisas com metodologia mais detalhada, do ponto de vista de avaliação econômica, realizadas sobre o tema, pode-se destacar uma coorte retrospectiva no Texas, EUA, de 1997-2008, com uma análise incluindo custos diretos e indiretos para vários serviços médicos. O custo médio por paciente do absenteísmo foi de US $\$ 196,00$ para o ano de $2008^{\mathbf{4 2}}$. No Brasil, são raros os trabalhos que indicam valores desperdiçados com o absenteísmo, destacando-se uma pesquisa realizada em um hospital público de Uberlândia, que identificou prejuízo, em 2011, de R\$ 1,1 milhão por ano, computando os procedimentos não realizados e o seu valor na Tabela SUS ${ }^{43}$.

O conhecimento dos dados sobre o absenteísmo na RSM-ES disponibilizados pelo Sesa-ES só foi possível devido à existência do SisReg-ES. O registro dos dados no sistema é passível de falhas, e não permite identificar se o absenteísmo ocorreu somente por problemas relacionados com o usuário ou se também foi agravado por questões de gestão. A Sesa-ES já identificou como problema os dados superestimados, considerando que, em alguns locais executantes, não é dada baixa no sistema quando o usuário é atendido, confirmando a realização do procedimento, o que compromete os dados encontrados, já que ficam computados como absenteísmo procedimentos realizados ${ }^{33}$.

Considerando as falhas recorrentes no registro dos atendimentos, nos diversos locais executantes, incluindo os serviços próprios, a Sesa-ES adotou medidas administrativas para atender à necessidade de adequação do Registro (chave de agendamento) de todos os usuários SUS, atendidos via SisReg, publicando em 20 de novembro de 2018, no Diário Oficial, a Portaria $\mathrm{n}^{\mathrm{0}} 084-\mathrm{R} / 2018$, que normatiza a baixa obrigatória no SisReg dos procedimentos realizados, que devem ser atualizados diariamente. Pretende-se, com esta medida, obter dados mais próximos do real sobre a atenção ao usuário.

São limitações deste estudo a fragilidade do banco de dados gerado no SisReg-ES, em função do não registro no sistema dos atendimentos realizados, gerando uma taxa maior 
de absenteísmo. Outra limitação foi a falta de análise econômica por local executante, de modo que não oferece informações sobre $o$ custo real por procedimento. Considerando os dados disponíveis, não é possível afirmar que todos os valores são realmente desperdiçados, devido às particularidades no desembolso monetário por serviços prestados nas diferentes modalidades de gestão na RSM-ES (serviços públicos estadual e federal, serviços filantrópicos, serviços privados e serviços públicos com gestão de OS). O custo estratificado por local daria ao estudo um valor do desperdício mais próximo do real.

As causas do absenteísmo são muito variadas e estão relacionadas com a gestão, com o usuário e com o trabalhador. Entretanto, um estudo realizado na atenção especializada na Espanha analisou essas causas, concluindo que o percentual de causas evitáveis é de $52,4 \%$. Isso sugere que haja amplo espaço para a implementação de esforços de correção. Justifica-se, portanto, uma análise individualizada futura do comportamento do absenteísmo em cada especialidade para subsidiar esses esforços.

\section{Conclusões}

O absenteísmo de usuários é um problema crônico nos sistemas de saúde no Brasil e no mundo, que pode inviabilizar a sustentabilidade da ampliação da oferta de serviços especializados. Na atenção especializada, ele é crescente na RSM-ES, com prejuízos para a gestão pública e para os usuários, levando em consideração, principalmente, o prejuízo social que ele acarreta. Salienta-se que as taxas de absenteísmo e os valores monetários encontrados representam dados significativos para o sistema de saúde pública.
Sugere-se que sejam buscadas as causas do absenteísmo relacionadas com a gestão e as particularidades do usuário, a fim de propor medidas de redução viáveis, tais como: fortalecimento da atenção básica como porta de entrada do usuário, qualificação do sistema de regulação, estabelecimento de redes de atenção à saúde regionalizadas e hierarquizadas e outras. Considera-se a integralidade da atenção como um dos maiores desafios do SUS, que exigem medidas inovadoras e capazes de promover cada vez mais um atendimento mais humanizado e comprometido com a vida.

Os dados de custos por serviço são importantes informações que ajudam na tomada de decisão por gestores, sobre investimentos e prioridades, apoiando a maior eficiência nos gastos em saúde. Assim, pode-se concluir que otimizar os recursos disponíveis e combater o desperdício sem comprometer a qualidade da assistência serão um desafio constante na agenda dos gestores do SUS na atualidade e nos próximos anos.

\section{Colaboradores}

Beltrame SM (0000-0002-0755-5565)* contribuiu para concepção, planejamento, metodologia e redação final. Oliveira AE (0000-0001-5160$3280) *$ contribuiu para concepção, planejamento, análise e interpretação dos dados, elaboração e revisão crítica da versão preliminar e da versão final do manuscrito. Santos MAB (0000-00027547-3537)* contribuiu para concepção, análise, elaboração do texto e revisão do documento final. Santos Neto ET (0000-0002-7351-7719)* contribuiu para concepção, planejamento, análise e interpretação dos dados, elaboração e revisão crítica da versão preliminar e da versão final do manuscrito. 


\section{Referências}

1. Santos JS. Absenteísmo dos usuários em consultas e procedimentos especializados agendados no SUS: um estudo em um município baiano. Vitória da Conquista. Dissertação [dissertação]. Salvador: Universidade Federal da Bahia; 2008; 33 p.

2. Bender ADS, Molina LR, Mello ALSFD. Absenteísmo na atenção secundária e suas implicações na atenção básica. Espaç Saúde (Online). 2010; 11(2):56-65.

3. Cavalcanti RP, Cavalcanti JCM, Serrano RMSM, et al. Absenteísmo de consultas especializadas nos sistemas de saúde público: relação entre causas e o processo de trabalho de equipes de saúde da família, João Pessoa - PB, Brasil. Rev Tempus Actas Saúde Col. 2013; 7(2):63-84.

4. Ellis DA, Jenkins R. Weekday affects attendance rate for medical appointments: large-scale data analysis and implications. PLoS ONE. 2012; 7(12):e51365.

5. Giunta D, Briatore A, Baum A, et al. Factors associated with nonattendance at clinical medicine scheduled outpatient appointments in a university general hospital. Patient PreferAdherence. 2013; 7:1163-70.

6. Jabalera Mesa ML, Morales Asencio JM, Rivas Ruiz F. Determinants and economic cost of patient absenteeism in outpatient departments of the Costa del Sol Health Agency. An Sist Sanit Navar. 2015; 38(2):235-45.

7. Dantas LF, Fleck JL, Cyrino Oliveira FL, et al. No-shows in appointment scheduling: a systematic literature review. Health Policy. 2018; 122(4):412-21.

8. Oleskovicz M, Oliva FL, Hildebrand e Grisi C, et al. Técnica de overbooking no atendimento público ambulatorial em uma unidade do Sistema Único de Saúde. Cad. Saúde Pública. 2014; 30(5):1009-17.

9. Bittar OJNV, Magalhães A, Martines CM, et al. Absenteísmo em atendimento ambulatorial de especialidades no estado de São Paulo. BEPA. 2016; 13(152):19-32.
10. Izecksohn MMV, Ferreira JT. Falta às consultas médicas agendadas: percepções dos usuários acompanhados pela Estratégia de Saúde da Família, Manguinhos, Rio de Janeiro. Rev Bras Med Fam Comunidade. 2014; 9(32):235-41.

11. Ferreira MB, Lopes AC, Lion MT, et al. Absenteísmo em consultas odontológicas programáticas na estratégia de saúde da família. Rev Univ Vale do Rio Verde. 2016; 14(1):411-9.

12. Gonçalves CÂ, Vazquez FL, Ambrosano GMB, et al. Estratégias para o enfrentamento do absenteísmo em consultas odontológicas nas Unidades de Saúde da Família de um município de grande porte: uma pesquisa-ação. Ciênc. Saúde Colet. 2015; 20(2):449-60.

13. Gurol-Urganci I, Jongh T, Vodopivec-Jamsek V, et al. Mobile phone messaging reminders for attendance at healthcare appointments. Cochrane Database Syst Rev. 2013; (12):CD007458.

14. Stubbs ND, Geraci SA, Stephenson PL, et al. Methods to reduce outpatient non-attendance. Am J Med Sci. 2012; 344(3):211-9.

15. Bech M. The economy of non-attendance and the expected effect of collecting a fine from non-participants. Health Policy. 2005; 74(2):181-91.

16. Mbada CE, Ajayi O, Agbeja OB, et al. Non-attendance for out-patient physiotherapy: evaluation, prediction and physiotherapists perceptions (a cross-sectional study). J Phys Ther. 2013; 7:12-22.

17. Mendes EV. As redes de atenção à saúde. 2. ed. Brasília, DF: OPAS; 2011.

18. Barreto ML, Teixeira MG, Bastos FI. Successes and failures in the control of infectious diseases in Brazil: social and environmental context, policies, interventions, and research needs. The Lancet. 2011; 377(9780):1877-89. 
19. Mendes EV. As redes de atenção à saúde. Ciênc. Saúde Colet. 2010; 15(5):2297-305.

20. Campos GWS. SUS: o que e como fazer? Ciênc. Saúde Colet. 2018; 23(6):1707-14.

21. Paim JS. Sistema Único de Saúde (SUS) aos 30 anos. Ciênc. Saúde Coletiva. 2018; 23(6):1723-8.

22. Spedo SM, Pinto NRS, Tanaka OY. O difícil acesso a serviços de média complexidade do SUS: o caso da cidade de São Paulo, Brasil. Physis. 2010; 20(3):95372 .

23. Duncan BB, Chor D, Aquino EML, et al. Doenças crônicas não transmissíveis no Brasil: prioridade para enfrentamento e investigação. Rev Saúde Pública. 2012; 46(supl1):126-34.

24. Schmidt MI, Duncan BB, Azevedo e Silva G, et al. Chronic non-communicable diseases in Brazil: burden and current challenges. Lancet. 2011; 377(9781):194961.

25. Souza MFM, Malta DC, França EB, et al. Transição da saúde e da doença no Brasil e nas Unidades Federadas durante os 30 anos do Sistema Único de Saúde. Ciênc. Saúde Colet. 2018; 23(6):1737-50.

26. Brasil. Ministério da Saúde. Portaria $n^{\circ} 1.559$, de $1^{\circ}$ de agosto de 2008. Institui a Política Nacional de Regulação do Sistema Único de Saúde (SUS). Diário Oficial da União [internet]. 4 Ago 2008 [acesso em 2019 nov 4]. Disponível em: http://www.saude.mt.gov.br/upload/legislacao/1559-\%5B2870-120110-SES-MT\%5D. pdf.

27. Vilarins GCM, Shimizu HE, Gutierrez MMU. A regulação em saúde: aspectos conceituais e operacionais. Saúde debate. 2012; 36(95):640-7.

28. Ferreira B. Faltas em consultas médicas pelo SUS chegam a $46 \%$ no ES [internet]. 2015 set 14 [acesso em 2018 ago 8]. Disponível em: https://blog.atencaobasica.org.br/2015/09/14/faltas-em-consultas-medicas-pelo-sus-chegam-a-46-no-es/.
29. Garcia LP. A economia desumana: porque mata a austeridade. Cad. Saúde Pública. 2016; 32(11):e00151116.

30. Holst J, Giovanella L, Andrade GCL. Porque não instituir copagamento no Sistema Único de Saúde: efeitos nocivos para o acesso a serviços e a saúde dos cidadãos. Saúde debate. 2016; 40(esp):213-26.

31. Silva EN, Silva MT, Pereira MG. Estudos de avaliação econômica em saúde: definição e aplicabilidade aos sistemas e serviços de saúde. Epidemiol Serv Saúde. 2016; 25(1):205-7.

32. Instituto Brasileiro de Geografia e Estatística [internet]. Censo 2010 [acesso em 2018 ago 8]. Disponível em: https://censo2010.ibge.gov.br/.

33. Espírito Santo. Secretaria de Estado da Saúde [internet]. Plano Diretor de Regionalização (PDR) [acesso em 2018 ago 8]. Disponível em: https://saude. es.gov.br/Media/sesa/SISPACTO/PDR-Plano\%20 Diretor\%20de\%20Regionaliza\%C3\%A7\%C3\%A3o_ ES_2011.pdf.

34. Lima LD, Viana ALÁ, Machado CV, et al. Regionalização e acesso à saúde nos estados brasileiros: condicionantes históricos e político-institucionais. Ciênc. Saúde Colet. 2012; 17(11):2881-92.

35. Giovanella L, Lobato LVC, Carvalho AI, et al. Sistemas Municipais de Saúde e a diretriz da integralidade da atenção: critérios para avaliação. Saúde debate. 2002; 26(60):37-61.

36. Pinheiro R, Guizardi FL. Cuidado e integralidade: por uma genealogia de saberes e práticas no cotidiano. In: Pinheiro R, Mattos RA, organizadores. Cuidado: as fronteiras da integralidade. São Paulo: Hucitec; 2003. p. 21-36.

37. Göttems LBD, Pires MRGM. Para além da atenção básica: reorganização do SUS por meio da interseção do setor político com o econômico. Saúde Soc. 2009; 18(2):189-98.

38. Mendes EV. O acesso à atenção primária à saúde. Brasília, DF: Conass; 2016. 
39. Sanchez RM, Ciconelli RM. Conceitos de acesso à saúde. Rev Panam Salud Pública. 2012; 31(3):260-8.

40. Tesser CD, Poli Neto P. Atenção especializada ambulatorial no Sistema Único de Saúde: para superar um vazio. Ciênc. Saúde Colet. 2017; 22(3):941-51.

41. Solla J, Chioro A. Atenção ambulatorial especializada. In: Giovanella L, Escorel S, Lobato LVC, et al., organizadores. Políticas e sistemas de saúde no Brasil. 2. ed. Rio de Janeiro: Fiocruz; 2012. p. 547-76.

42. Kheirkhah P, Feng Q, Travis ML, et al. Prevalence, predictors and economic consequences of no-shows. BMC Health Serv Res. 2016; 16:13.
43. Fernandes A. Uma em quatro pessoas não vai à consulta [internet]. [acesso em 2019 ago 8]. Disponível em: http://www.correiodeuberlandia.com.br/cidade-e-regiao/uma-a-cada-4-pessoasnao-vai-as-consultas/.

Recebido em 26/04/2019

Aprovado em 22/10/2019

Conflito de interesses: inexistente

Suporte financeiro: Fundação de Amparo à Pesquisa e Inovação do Espírito Santo (Fapes) 